\title{
Component-Based Construction of Real-Time Systems in BIP
}

\author{
Joseph Sifakis \\ Verimag
}

BIP is a framework for the component-based construction of real-time systems. It considers that systems can be obtained as the composition of 3-layer components. For a component,

- The lower layer describes its behavior, a set of transitions with triggers and actions (atomic state transformations). A trigger consists of an enabling condition on data and a port through which synchronization is sought.

- The intermediate level is the set of interactions between transitions of the behavior level. An interaction is a set of synchronizing ports and associated actions. Interactions are specified by using connectors expressing synchronization constraints on ports.

- The upper level is a set of priority rules implementing scheduling policies for interactions.

The framework supports a system construction methodology which is based on a parameterized binary composition operator on components. The product of two components consists in composing their corresponding layers, separately. Parameters are used to define new interactions as well as new priorities between the composed components.

Composition of components is defined by operational semantics. Behaviors are transition systems labeled with interactions. Interaction models and priority rules are behavior transformers.

The use of a layered composition operator allows incremental construction, that is any compound component can be obtained by successive composition of its constituents.

The system construction process in BIP can be considered as a sequence of transformations in a three-dimensional space: Behavior, Interaction, Priority. Elements of the interaction $\times$ priority space characterize the overall architecture. A transformation is the result of the superposition of elementary transformations for each dimension. This provides a basis for the study of correct-by-construction system transformations, in particular for the following:

- Architecture transformations allowing preservation of properties of the underlying behavior. Such transformations can provide (sufficient) conditions for compositionality and composability results.

- Transformations allowing to obtain from a BIP model an observationally equivalent distributed implementation. These involve displacement along the

A. Bouajjani and O. Maler (Eds.): CAV 2009, LNCS 5643, pp. 33-34, 2009.

(C) Springer-Verlag Berlin Heidelberg 2009 
three coordinates. They consist in replacing multiparty interaction and priorities by protocols based on asynchronous message passing.

The BIP framework has been implemented in a language and a toolset. The BIP language offers primitives and constructs for modeling and composing atomic components described as state machines, extended with data and functions in C. The BIP toolset includes an editor and a compiler for generating from BIP programs, $\mathrm{C}++$ code executable on a dedicated platform. It allows simulation and verification of BIP programs by model checking or by compositional verification techniques for state invariants and deadlock-freedom.

We provide two examples illustrating the use of BIP for modeling and verifying heterogeneous systems: an MPEG encoder, and a hierarchical robotic system.

For further information: http://www-verimag.imag.fr/ async/bip.html 